\title{
An Intelligent Anti-Money Laundering Method for Detecting Risky Users in the Banking Systems
}

\author{
Neda Heidarinia \\ Islamic Azad University \\ Science and Research Branch \\ Dept. of Computer Engineering \\ Neyshabur, Iran
}

\author{
Ali Harounabadi \\ Islamic Azad University \\ Central Tehran Branch \\ Dept. of Computer Engineering \\ Tehran, Iran
}

\author{
Mehdi Sadeghzadeh \\ Islamic Azad University \\ Mahshahr Branch \\ Dept. of Computer Engineering \\ Mahshahr, Iran
}

\begin{abstract}
During the last decades, universal economy has experienced money laundering and its destructive impact on the economy of the countries. Money laundering is the process of converting or transferring an asset in order to conceal its illegal source or assist someone that is involved in such crimes. Criminals generally attempt to clean the sources of the funds obtained by crime, using the banking system. Due to the large amount of information in the banks, detecting such behaviors is not feasible without anti-money laundering systems. Money laundering detection is one of the areas, where data mining tools can be useful and effective.

In this research, some of the features of the users are extracted from their profiles by studying them. These features may include large financial transactions in risky areas regarding money laundering, reactivation of dormant accounts with considerable amounts, etc. Network training is performed by designing a fuzzy system, developing an adaptive neuro-fuzzy inference system and adding feature vectors of the users to it. The network output can determine the riskiness of the user behavior. The evaluation results reveal that the proposed method increases the accuracy of detecting risky users.
\end{abstract}

\section{Keywords}

Money laundering, Neuro-Fuzzy, ANFIS

\section{INTRODUCTION}

Money laundering is a long, continuous, complex, and collective process that is generally executed in large scale. It transforms the dirty money obtained by crime into legitimate money, so that it is difficult to track its criminal source. Intelligent criminal behaviors, frequent behavior changes by the criminals and their advantage over the inspectors are some of the most important reasons that justify the need for a powerful intelligent tool in order to detect and report suspicious criminal behaviors inside the large amount of data regarding customer behavior.

Nowadays, data is collected and recorded rapidly in most organizations, but it is not easy to use this data in most cases. The data is analyzed through data mining to extract the possible trends, inconspicuous connections and hidden patterns from the large amount of data.

Recently, the combination of artificial neural networks and fuzzy logic, which is called fuzzy neural network, is applied frequently as a nonlinear estimation tool. It has many advantages in prediction, classification, clustering and optimization techniques.

\section{RELATED WORK}

Money laundering is a serious crime which makes it necessary to develop detection methods in transactions. Some researches have been carried on, but the problem is not thoroughly solved. From reference [1], we can see that antimoney laundering issue is much more obvious and needs urgent settlement. Several detecting technologies nowadays have been applied in anti-money laundering field are proposed, in [2,3], decision tree classification and outlier analysis tools were introduced in extracting suspicious financial transaction. And in $[4,5,6]$, such as classification analysis tools (Bayesian classification, Genetic algorithms etc), cluster analysis tools, support vector machine(SVM), case-based reasoning, rough set, link analysis, intelligent agents and neural network were mentioned. Outlier detection is used to discover data object which is not consistent with the features of general data, but it can't recognize the suspicious behavior of peer comparison. SVM can get good result, but the time cost is high. In [7], a radial basis function (RBF) neural network model based on APC-III clustering algorithm and recursive least square algorithm is proposed for antimoney laundering. APC-III clustering algorithm is used for determining the parameters of radial basis function in hidden layer, and recursive least square (RLS) algorithm is adopted to update weights of connections between hidden layer and output layer.

In [8], a new machine learning method is proposed for mining transaction graphs. This method generates a model from a graph that might include suspicious transaction. This model is parameterized using fuzzy numbers, which represent the parameters of the transactions and the graphs of the discovered transactions.

Data mining methods are among other methods of crime detection. They focus on statistical analyses, discovering customer behavior and patterns to detect crimes [9]. These methods are based on learning particular rules and they can extract fraudulent behavior indexes from large customer transaction databases. These indexes are used to create monitoring systems in order to record unusual behaviors of the customers and discover the suspicious ones among them. The output of these systems can be used to issue warnings about wrongdoer users [10].

\section{PROPOSED METHOD}

Due to the importance of money laundering, a new method is proposed in this research to detect the suspicious accounts. By combining artificial neural networks and fuzzy logic, a fuzzy system is implemented that is capable of training. It functions as follows; while moving forward in each epoch, the node 
outputs are calculated normally until the last layer. Then, the resulting parameters are calculated by least squares error. While moving backwards after calculating the error, it is propagated among condition parameters and their values are adjusted using gradient descent. Various structures are proposed to implement a fuzzy system with neural networks. Adaptive neuro-fuzzy inference system (ANFIS) is one of the most powerful ones that was invented by Jaris $[11,12]$.

\subsection{Details of the Proposed Structure}

According to discussions above, it was concluded that hybrid methods are the best choice for large datasets, since they have high accuracy and acceptable speed. The proposed method is based on ANFIS because of its appropriate capabilities and attributes.

Adaptive neuro-fuzzy inference system applies neural network and fuzzy logic algorithms to design the nonlinear mapping between the input and output domains. With linguistic power of fuzzy systems and numeral power of neural networks, ANFIS is very powerful in modelling complex processes [13]. Figure1 presents the general structure of the proposed method.

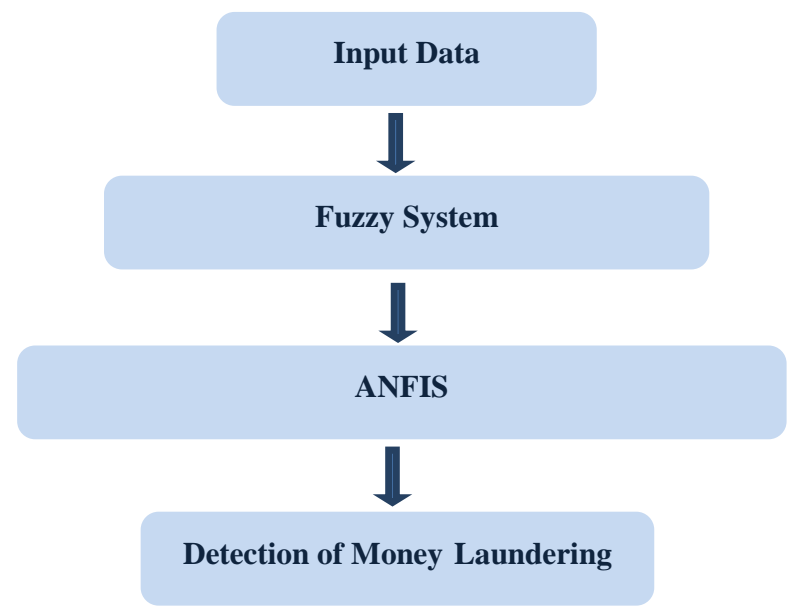

Fig 1: The general structure of the proposed method

The data is extracted from the datasets of the bank and the fuzzy system is designed based on the features of this data and some fuzzy rules. It detects money laundering intelligently using the optimal patterns produced in an ANFIS system.

\subsubsection{Fuzzy System and Extracted User Features}

Since it is not always possible to discuss the results of a problem definitely, some methods are required for providing approximate or fuzzy descriptions of a model, which are both acceptable and analyzable. In this research, the feature vector of the users is created based on their bank account history. The riskiness of the users can be determined by tracking their accounts and focusing on the following items:

- $\quad$ Large and frequent deposits and withdrawals during a limited period

- Large financial transactions in risky areas regarding money laundering (in free trade-industrial zones)

- Reactivation of dormant accounts with considerable amounts

- Frequent operations and transactions of less than a specified threshold amount (during a limited period) in order to evade the regulations of money laundering prevention
- Frequent send and receive transactions among several accounts during a short period of time

When the ANFIS network is trained for various users, it can be used to determine the riskiness of the users in real-time through network output.

In this research, five fuzzy input parameters are used for the aforementioned items, as follows:

- Standard deviation of the account

- $\quad$ Free zones

- The interval between transactions

- Frequent operations of less than the permitted threshold

- Frequent send and receive transactions of the account

Sugeno inference engine is selected as the core of the proposed fuzzy system.

Generally, Sugeno-type systems can be used to model inference systems with linear and constant output membership functions. Generally, because of their compact representation and higher computational performance compared to Mamdani systems, Sugeno systems are used for applying adaptive techniques to create fuzzy models. These adaptive techniques can be used to customize the membership functions.

\subsubsection{The ANFIS Model}

ANFIS is a 5-layered network composed of nodes and links. The first layer includes the input data and the membership degrees defined by the user. All of the modelling operations are performed in layers 2 to 4 . Network output is the last layer, which is responsible for minimizing the difference between the output obtained from the network and the real one.

The main training method of ANFIS is error backpropagation. In this method, the error is propagated toward the inputs and the parameters are adjusted using the steepest descent algorithm. After error propagation, the training algorithm continues as follows. The network produces an output $Z_{k}$ (or a series of outputs) for a given input pattern and compares this result with the desired reaction of each neuron $d_{k}$. Then, network weights are adjusted to remove or reduce the error and the next pattern is obtained. The process of weight adjustment continues until all of the errors are less than the predefined tolerance level [14]. The reason why all these updates gradually result in minimizing mean squared error (MSE) is that all of the input patterns rely on the fact that the error function of the training algorithm has a descending gradient after error propagation.

The purpose of training is to adjust the weights in a way that the desired outputs are obtained when the network is provided with a series of inputs. Therefore, the network is generally trained with a large number of input-output pairs, which are called samples.

The training method is as follows:

- $\quad$ Selecting small random numbers (both negative and positive) as the weights

- An input-output training pair is selected from the training set

- The input vector is fed to the network and the output is calculated

- The error (the difference between the network output and the desired one) is calculated

- The network weights are adjusted in a way that the error is minimized 
- Steps 2 to 5 are repeated for each of the training pairs in the training set until the error is acceptable [15].

Sub clustering is the selection method of the adaptive neurofuzzy network. The training method includes a combination of least squares and back propagation.

\subsection{The Advantages of ANFIS}

The main advantages of the adaptive neuro-fuzzy inference system, compared to the other systems, can be summarized as follows:

- Applying neural networks for data arrangement and pattern detection.

- Creating a transparent fuzzy inference system that has few drawbacks and produces less calculation error compared to the other neural networks.

- This system retains the advantages of fuzzy expert systems, while reducing the need for an expert.

- Due to the application of fuzzy logic in this method, the problems of modelling and analyzing complex data are reduced.

- This system can process the qualitative aspects of human experience.

- The neuro-fuzzy system includes training capabilities while retaining the advantages of the fuzzy inference system [16].

- Unlike other systems based on fuzzy logic, there is no need to convert crisp data to fuzzy data and initialize the parameters.

\section{EXPERIMENT}

\subsection{Experiment Data Set}

In this research, the statistical population under study includes the accounts of the bank customers. Due to the restrictions and obstacles of data collection and confidentiality of the information, access to the real dataset of customer transactions was denied. Therefore, a hypothetical dataset was generated based on the real dataset of the bank.

As it was discussed earlier, a feature vector is considered for each user based on his/her bank account history. After studying and analyzing the aforementioned features for suspicious accounts and with the help of the bank experts, some parameters were extracted through various calculations, which were not necessarily considered in the initial tables. After the final analysis, five parameters that played a role in determining the account type were selected as the input variables and a parameter was assigned as the output variable in the system. These parameters are described in table1. The dataset consists of 2000 records concerning the customer accounts. Each record contains the information presented in table 1.
Table 1. The parameters used in the detection of money laundering

\begin{tabular}{|c|c|c|}
\hline & Parameter name & $\begin{array}{c}\text { Parameter type } \\
\text { Input/Output }\end{array}$ \\
\hline 1 & Standard deviation of the account & Input \\
\hline 2 & Free zones & Input \\
\hline 3 & The interval between transactions & Input \\
\hline 4 & $\begin{array}{c}\text { Operations of less than the } \\
\text { permitted threshold }\end{array}$ & Input \\
\hline 5 & $\begin{array}{c}\text { Send and receive transactions of } \\
\text { the account }\end{array}$ & Input \\
\hline 6 & Account type & Output \\
\hline
\end{tabular}

In the aforementioned dataset, standard deviation of the account was considered for the feature regarding deposit and withdrawal amounts. There were 200 records concerning free zone transactions. An interval ranging from 1 day to 365 days was considered between transactions as the inactivity period of an account. Operation count was assumed to be between 3 and 800 for the feature regarding the frequent operations and transactions of less than a specified threshold amount during a certain period. The transaction count was considered to vary between 3 and 600 for the feature concerning frequent send and receive transactions among several accounts.

\subsection{Experiment Analysis}

The data is divided into train and test sets, each containing 1500 and 500 records, respectively. First, the fuzzy inference system, the inputs, their range and membership functions are defined. Their range determines the number of membership functions. The domain and range of the membership functions define their type. In this system, most of the functions are Gaussian. A fuzzy inference system is created using subtractive clustering method that classifies inputs and outputs at different levels. Rule database is the main part of a fuzzy system. In this system, 48 rules are extracted. Since decision-making is performed by checking all of the rules in fuzzy inference systems, their truth values must be combined in some way.

Finally, the resulting fuzzy set must be defuzzificated. ANFIS improves the initial fuzzy inference system by training it. In the end, it performs the final account detection.

Tables 2 and 3 show real and predicted amounts of the output in two cases of training and testing. As an example in table 2, one account has a high standard deviation and its transaction has been operated in free zones. Number of operations being less than the permitted threshold is a lot, for this account with many send and receive transactions with other accounts. Hence, this account is considered suspicious. In fact its output must be 1 , stating that the amount of the predictable output in the case of training is close to the amount of the real output. Similar situation applies to the case of testing. 
Table 2. The real and predicted amounts of the output in the case of training

\begin{tabular}{|c|c|c|c|c|}
\hline $\begin{array}{c}\text { Standard } \\
\text { deviation of } \\
\text { the account }\end{array}$ & $\begin{array}{c}\text { Operations } \\
\text { of less than } \\
\text { the } \\
\text { permitted } \\
\text { threshold }\end{array}$ & $\begin{array}{c}\text { Send and } \\
\text { receive } \\
\text { transactions } \\
\text { of the } \\
\text { account }\end{array}$ & $\begin{array}{c}\text { The } \\
\text { real } \\
\text { amount } \\
\text { of the } \\
\text { output }\end{array}$ & $\begin{array}{c}\text { Predicted } \\
\text { amount } \\
\text { of the } \\
\text { output in } \\
\text { the case } \\
\text { of } \\
\text { training }\end{array}$ \\
\hline 386.9193045 & 771 & 588 & 1 & 1.0876694 \\
\hline 1224.235418 & 765 & 114 & 1 & 1.1120187 \\
\hline 753.4890929 & 183 & 525 & 1 & 0.966794 \\
\hline 453.8909506 & 582 & 162 & 0 & 0.0017701 \\
\hline 2121.910616 & 51 & 126 & 0 & -0.012072 \\
\hline
\end{tabular}

Table 3. The real and predicted amounts of the output in the case of testing

\begin{tabular}{|c|c|c|c|c|}
\hline $\begin{array}{c}\text { Standard } \\
\text { deviation of } \\
\text { the account }\end{array}$ & $\begin{array}{c}\text { Operations } \\
\text { of less than } \\
\text { the } \\
\text { permitted } \\
\text { threshold }\end{array}$ & $\begin{array}{c}\text { Send and } \\
\text { receive } \\
\text { transactions } \\
\text { of the } \\
\text { account }\end{array}$ & $\begin{array}{c}\text { The } \\
\text { real } \\
\text { amount } \\
\text { of the } \\
\text { output }\end{array}$ & $\begin{array}{c}\text { Predicted } \\
\text { amount } \\
\text { of the } \\
\text { output in } \\
\text { the case } \\
\text { of testing }\end{array}$ \\
\hline 236.0157467 & 447 & 474 & 1 & 1.0366976 \\
\hline 2161.78315 & 681 & 342 & 1 & 0.9797137 \\
\hline 424.8703517 & 486 & 387 & 1 & 1.0023441 \\
\hline 368.1250191 & 255 & 60 & 0 & -0.110064 \\
\hline 1026.043835 & 84 & 384 & 0 & -0.093818 \\
\hline
\end{tabular}

The following diagrams present different system evaluations.

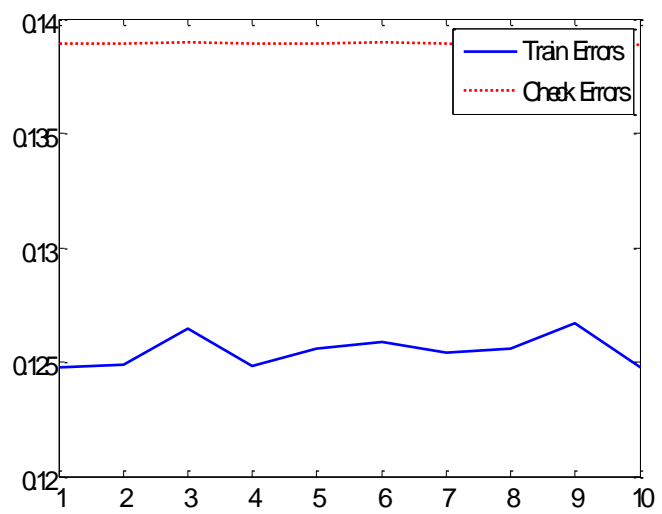

Diagram 1. Estimation Error

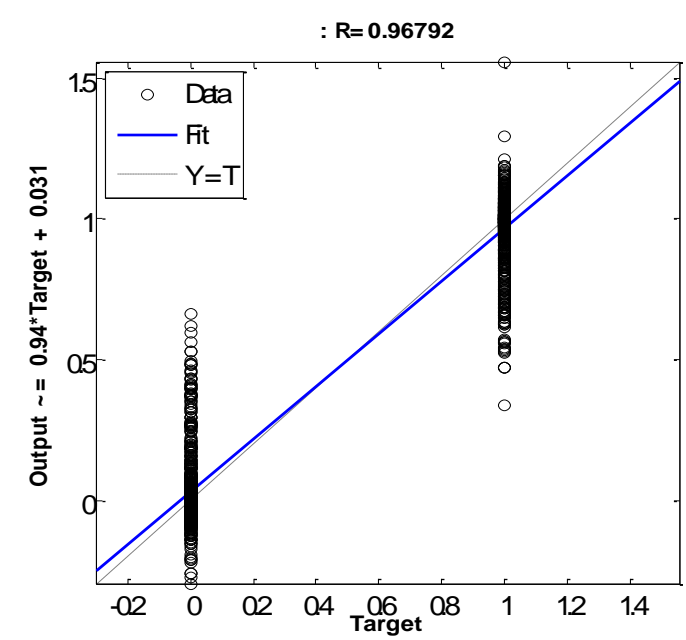

Diagram 2. Training Data Regression

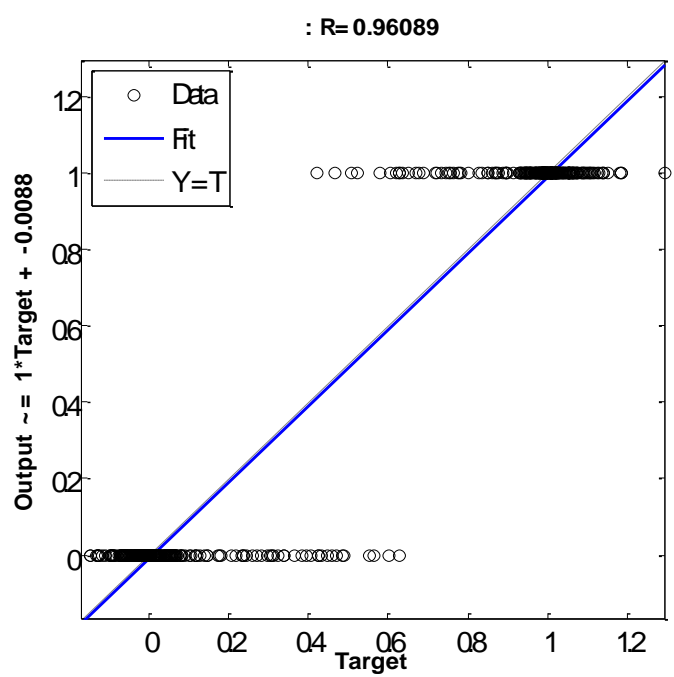

Diagram 3. Training Data Regression

\section{CONCLUSION}

This research introduced a new and intelligent anti-money laundering method for suspicious account detection. ANFIS was applied to design a system for detecting suspicious accounts and transactions. The system was implemented using MATLAB software application.

A fuzzy system was created as the input of ANFIS, using sub clustering method. Customer's behavior regarding the account was considered as the system input, which included 5 features. ANFIS trained and improved the initial fuzzy system. Finally, the trained network was applied to test and detect the suspicious accounts. During training phase, the difference between the output and real values were reduced by adjusting the membership degree parameters based on the acceptable error. The resulting detection accuracy of the proposed system was $96 \%$.

Increasing the accuracy of suspicious account detection was one of the results of this research. 


\section{REFERENCES}

[1] Qifeng, Y. Bin, F. Ping, S. Sept. 2007. Study on AntiMoney Laundering Service System of Online Payment based on Union-Bank mode. International Conference on Wireless Communications, Networking and Mobile Computing. pp: 4991-4994.

[2] TIANQING, Z. Dec.2006. An Outlier Detection Model Based on Cross Datasets Comparison for Financial Surveillance. Proceedings of the 2006 IEEE Asia-Pacific Conference on Services Computing (APSCC'06), pp: 601-604.

[3] WANG, S. YANG, J. August 2007. A Money Laundering Risk Evaluation Method Based on Decision Tree. Proceedings of the Sixth International Conference on Machine Learning and Cybernetics, Hong Kong.

[4] Xuan, L. Pengzhu, Z. Sept.2007. An Agent based AntiMoney Laundering System Architecture for Financial Supervision. International Conference on Wireless Communications, Networking and Mobile Computing. pp: 472-5475.

[5] Gao, SH. Xu, D. 2007. Conceptual Modeling and Development of an Intelligent Agent-Assisted Decision Support System for Anti-Money Laundering. Expert System with Applications, doi:10.1016/j.eswa.

[6] Gao, SH. Xu, D. Wang, H. Wang, Y. 2006. Intelligent Anti-money Laundering System. International Conference on Service Operation and Loqistics, and Informatics, SOLI'06, IEEE. pp: 851-856.

[7] LV , L.T. , JI , N. , ZHANG, J.L. , 2008. A RBF Neural Network Model for Anti-Money Laundering. Proceedings of the International Conference on Wavelet Analysis and Pattern Recognition. IEEE , PP:209-215.
[8] Michalak, K. , Korczak, J. 2011. Graph Mining Approach to Suspicious Transaction Detection. Proceedings of the Federated Conference on Computer Science and Information Systems. IEEE , PP:69-75.

[9] Quah, J. T. S, Sriganesh, M. 2007. Real-Time Credit Card Fraud Detection using Computational Intelligence. Expert System swith Applications, pp.9-17.

[10] Fang, L. Cai, M. Fu, H. Dong, J. 2007. OntologyBased Fraud Detection. in Computational Science. pp:1048-1055 .

[11] Jang, J.R. Sun, C. 1995. Nero Fuzzy Modelling and Control. Proc. of the IEEE. PP: 378-405.

[12] Kuo, R.J. Chen, C.H. Hwang, Y.C. 2001. An Intelligent Stock Trading Decision Support System through Integration of Genetic Algorithm based Fuzzy Neural Network and Artificial Neural Network. Fuzzy Sets and Systems 118 .

[13] jang , J.S.R. 1993. ANFIS : Adaptive - Network based Fuzzy Inference Systems. Department of Electrical Engineering and Computer Science, University of California Berkeley.

[14] Parker, D.B. 1985. Learning Logic: Casting the Cortex of the Human Brain in Silicon. technical report TR-47. Cambridge, MA: Center for Computational Research in Economics and Management, MIT.

[15] Siler W. Buckley J.J. 2005. Fuzzy Expert Systems and Fuzzy Reasoning. New Jersey, Wiley Interscience, 54.

[16] Ata, R. Kocyigit, Y. 2010. An Adaptive Neuro-Fuzzy Inference System Approach for Prediction of Tip Speed Ratio in Wind Turbines. Expert Systems with Applications. pp: 5454-5460. 